\title{
Toughening mechanisms in thermoplastic-modified epoxies: 1. Modification using poly(phenylene oxide)
}

\author{
Raymond A. Pearson" and Albert F. Yee \\ Department of Materials Science and Engineering, University of Michigan, Ann Arbor, \\ Michigan 48109, USA
}

(Received 20 May 1992; revised 31 August 1992)

\begin{abstract}
An epoxy based on the diglycidyl ether of bisphenol A (DGEBA) has been modified with poly(phenylene oxide) (PPO) and cured with piperidine. A two-phase alloy resulted, in which the DGEBA epoxy was the continuous phase. Several PPO loadings were investigated. The tensile yield strengths of these PPO-modified epoxies were found to be independent of PPO content. In contrast, the fracture toughness improved with PPO content in a linear fashion. The micromechanical mechanism responsible for the improvement in toughness was found to consist of crack bifurcation and microcracking. Some evidence of particle bridging was also observed, and it is thought that particle bridging may play an important role in the formation of a microcracked damage zone.
\end{abstract}

(Keywords: epoxy resin; thermoplastic modification; toughening; poly(phenylene oxide); mechanical properties)

\section{INTRODUCTION}

The rubber modification of epoxy resins is a well known technology, which was invented to improve the fracture toughness of brittle epoxies. This technology originated in the late $1960 \mathrm{~s}^{1,2}$. Preliminary studies on rubbermodified epoxies reported only modest increases in fracture toughness; however, as the technology developed, more significant increases in toughness were obtained ${ }^{3-10}$. In more recent years, it has been shown that the toughness can be increased by more than an order of magnitude over that of the unmodified epoxy $y^{1-16}$. This progress can be attributed, at least in part, to the understanding of the influence of various material parameters such as crosslink density of the epoxy ${ }^{17-20}$, size of the rubber particles $^{21}$ and cohesive strength of the particle on toughness enhancement.

However, despite our improved understanding of rubber-modified epoxies, the drawbacks associated with this technology persist. For example, the addition of soft rubbery particles to an epoxy diminishes its elastic modulus, yield strength and creep resistance. Ordinarily, these compromises are tolerable since a significant increase in toughness is achieved. However, increases in fracture toughness are only observed for lightly crosslinked epoxies ${ }^{17-20}$. Rubber modification fails to produce a significant improvement in highly crosslinked systems; thus the utility of rubber-modifying highly crosslinked epoxies is questionable and is usually avoided. This is not a desirable situation since most of the advanced thermosetting resins for aerospace applications consist of highly crosslinked network

* To whom correspondence should be addressed, at present address: Department of Materials Science and Engineering, Lehigh University, Bethlehem, Pennsylvania 18015, USA polymers, which are often brittle. Fortunately, a new toughening technology has emerged that avoids the classic compromises associated with rubber toughening. This new technology utilizes another type of toughening phase: rigid, thermoplastic particles.

The use of rigid, thermoplastic particles to toughen epoxy is a relatively novel toughening approach. This technology originated in the early $1980 \mathrm{~s}^{22-28}$. Bucknall and Partridge $e^{22}$ appear to have been the first to publish on this subject. Unfortunately, the increases in fracture toughness found by these authors ${ }^{22}$ were small. However, significant progress in thermoplastic-toughened epoxies was achieved by researchers at Virginia Polytechnic Institute ${ }^{23,24}$, who have focused on the synthesis of polysulfone (PSF) oligomers to be used as toughening agents for epoxies. We should also mention that other researchers have contributed to the synthesis of thermoplastic-modified epoxies ${ }^{25-28}$. Yet, despite the significant progress made in synthesizing thermoplasticmodified epoxies, the fracture and toughening mechanisms in these modified epoxies are not well understood. Therefore, it is useful to begin our discussion on thermoplastic-modified epoxies by reviewing several toughening mechanisms that have been proposed for these novel materials. These mechanisms are illustrated in Figure 1.

\section{Toughening by the crack bridging mechanism}

Crack bridging is often invoked as a possible toughening mechanism for thermoplastic-modified epoxies. The proposed role of the rigid plastic particles is to span the two crack surfaces and apply surface tractions that effectively reduce the $K$ applied at the crack tip. An alternative explanation of toughening via crack bridging considers the energy consumed when the 


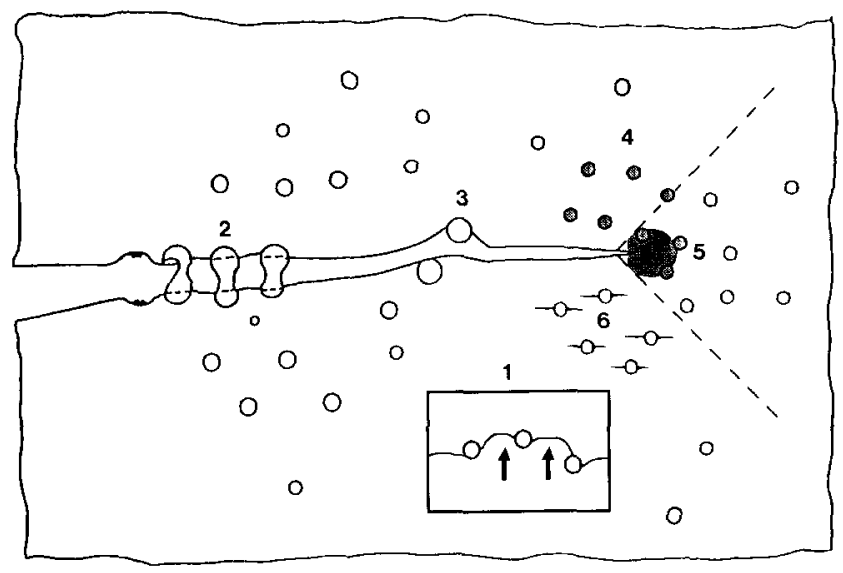

Figure 1 Schematic diagram of toughening mechanisms proposed for thermoplastic-modified epoxies: (1) crack pinning, (2) particle bridging, (3) crack path deflection, (4) particle yielding, (5) particle-yieldinginduced shear banding, and (6) microcracking

thermoplastic particles are plastically deformed and then torn. Physical evidence for the crack bridging mechanism has been inferred from examinations of fracture surfaces using scanning electron microscopy (SEM). In its simplest form, crack bridging consists of damaged particles on the plane of the crack; thus examination of the fracture surface by SEM would be useful.

The amount of toughness one can expect from the bridging of the crack by rigid thermoplastic particles is unclear. To estimate the amount of toughness one can expect, it is useful to examine crack bridging models from two other types of modified epoxies: rubber-modified and glass-filled.

A crack bridging model proposed by Ahmad, Ashby and Baumont ${ }^{29}$ emphasizes the strength of the rubber particles in rubber-modified epoxies. This model is based on the idea that improvements in toughness may be attributed solely to the stretching and tearing of rubber particles in the crack wake. To facilitate the amount of particle stretching, large particles should be used. The improvements in toughness can be rationalized by considering the shielding effect, which is the reduction of $K$ at the crack tip, when these particles apply compressive tractions in the crack wake. Quantitatively, the model may be stated as:

$$
\frac{K_{\mathrm{Ic}}}{K_{0}}=\phi(1-f)+\frac{f E^{*} \Gamma_{\mathrm{t}}}{2 \phi(1-f) K_{0}^{2}}
$$

where $K_{\mathrm{Ic}}$ is the fracture toughness of the rubber-modified epoxy, $K_{0}$ is the fracture toughness of the unmodified epoxy, $\phi$ is a correction factor that accounts for crack bowing, $f$ is the volume fraction of rubber particles, and $\Gamma_{t}$ is the tearing energy of the rubber particles.

The model predicts that the size of the particle affects the total tear energy consumed. In particular, improvements in toughness should be greater when larger particles are used. In addition to particle size, the model also considers the influence of the stiffness of the particle $\left(E^{*}\right)$ and the tear energy $\left(\Gamma_{t}\right)$; increases in particle stiffness and tear strength should result in an increase in crack shielding, hence toughness. Although the amount of toughening attributed to crack bridging has been shown to be negligible in rubber-modified epoxies, it could conceivably be a more dominant mechanism when rigid thermoplastic particles are used, since they are much more rigid and much stronger than elastomeric particles. Parenthetically speaking, we must remember that this particular bridging model has been developed for rubber-modified polymers, and it should not be applied to the fracture of thermoplastic-modified epoxies without modification.

A crack bridging model proposed by $\operatorname{Rose}^{30}$ for glass-filled epoxies emphasizes the ability of 'impenetrable' particles to bow the crack front and also the ability of these particles to act as springs to keep the two crack surfaces shut. This particular model attempts to explain the maxima often seen in fracture toughness when glass spheres are added in high concentrations to an epoxy resin. In brief, it has been proposed that the observed maxima in toughness (as a function of filler content) are due to the ease of circumventing the particle relative to matrix cracking. In essence, Rose's model is very similar to that of Ahmad et al., in that both models attempt to predict the size of the ligament zone behind the crack tip and relate this to the toughness measured. Quantitatively, the model may be stated as:

$$
\frac{K_{\mathrm{c}}}{K_{0}}=\frac{\left[(2 s / \lambda)+(2 r / \lambda)\left(K_{\mathrm{L}} / K_{0}\right)^{2}\right]^{1 / 2}}{F_{\mathbf{I}}}
$$

where $K_{\mathrm{c}}$ is the fracture toughness of the modified epoxy, $K_{0}$ is the fracture toughness of the unmodified epoxy, $2 s$ is the surface-to-surface obstacle spacing, $2 r$ is the diameter of the particles, $\lambda$ is the centre-to-centre obstacle spacing, $K_{\mathrm{L}}$ is a limiting stress intensity factor that specifies the failure of the trailing end of the reinforced zone, and $F_{\mathrm{I}}$ is an interpolating function constructed to reproduce the correct asymptotic expansions for soft springs and for hard springs.

Rose proposes that the $K_{\mathrm{L}} / K_{0}$ ratio can be used to characterize the ease of fracturing or circumventing the second phase relative to matrix cracking. Moreover, he proposes that the $K_{\mathrm{L}} / K_{0}$ ratio can be used as an adjustable parameter to fit experimental data. The good fit appears to support the validity of this model.

Evidence for crack bridging in thermoplastic-modified epoxies has been presented by several investigators ${ }^{23-27}$. The majority of these works consisted of a post-mortem analysis of fracture surfaces in which torn particles could be observed. Other evidence is indirect and only suggestive. For example, Cecere and $\mathrm{McGrath}^{24}$ showed that increasing the molecular weight of the thermoplastic phase improves the amount of toughness enhancement observed; high-molecular-weight polymers often display higher elongations to failure. Another example is given by Raghava ${ }^{25}$, who has shown that the brittle (low elongation to failure) poly(ether sulfone) (PES) does not work as a toughening agent for epoxies.

\section{Toughening by crack pinning}

Although not as often cited as crack bridging, toughening by crack pinning has been $\operatorname{cited}^{26}$ as the mechanism providing toughness in thermoplasticmodified epoxy. In this mechanism, the proposed role of the rigid thermoplastic particles is to behave as imprenetrable objects that cause the crack to bow out, which consumes extra energy. Indeed, the difference in toughness between the brittle epoxy and the ductile thermoplastic phase is large enough to consider these particles as relatively impenetrable. Indirect evidence for the occurrence of this mechanism is the observation of 
'tails' near the particles on the fracture surface when viewed using SEM. The amount of toughness one can expect from this mechanism with these particles is unclear, since researchers have modelled the process differently ${ }^{30-32}$, and, at present, most of the effort is focused on glass-filled systems, which are reviewed below.

The original concept of crack pinning was developed by Lange ${ }^{31}$, who showed that the crack front changed in length as it interacts with an inhomogeneous particle; thus more energy is required to propagate the crack. A schematic of the model is shown in the insert of Figure 1. The toughening effect due to this model is as follows:

$$
\frac{G_{\mathrm{Ic}}}{G_{0}}=1+\frac{T}{d_{\mathrm{s}} G_{0}}
$$

where $G_{I c}$ is the fracture toughness of the modified epoxy, $G_{0}$ is the fracture toughness of the unmodified epoxy, $T$ is the line energy per unit crack front (line tension), and $d_{\mathrm{s}}$ is the centre-to-centre distance between particles. The centre-to-centre distance between particles is calculated as follows:

$$
d_{\mathrm{s}}=\frac{2 d_{\mathrm{p}}(1-f)}{3 f}
$$

where $d_{\mathrm{p}}$ is the diameter of the particles and $f$ is the volume fraction of particles.

Evans $^{32}$ refined the original concept of crack pinning developed by Lange ${ }^{31}$ in an attempt to predict the non-linear relation between fracture toughness and the volume fraction of particles in the glass-filled epoxy data provided by Lange and Radford ${ }^{\mathbf{3 3}}$. Evans' refined model assumes the existence of semi-elliptical crack fronts between particles, which interact to lower the stress needed to propagate them. The model has the following formulation:

$$
\frac{K_{\mathrm{c}}}{K_{0}}=\left(\frac{\pi}{2+d_{\mathrm{p}} / C}\right)^{1 / 2}\left[\tan \left(\frac{\pi}{2+d_{\mathrm{p}} / C}\right)\right]^{1 / 2}
$$

where $K_{\mathrm{c}}$ is the fracture toughness of the modified epoxy, $K_{0}$ is the fracture toughness of the unmodified epoxy, $2 C$ is the surface-to-surface obstacle spacing, and $d_{\mathrm{p}}$ is the diameter of the particles.

Evans' correction for the interacting crack fronts appears to predict the non-linear increase in fracture toughness from the data of Lange and Radford ${ }^{33}$ reasonably well. However, the interactions between semi-elliptical crack fronts cannot explain the maximum displayed by the data. Evans attributes the maxima in the toughness data to the penetration of the crack into the particles.

As explained in the previous subsection, Rose $\mathrm{R}^{30}$ developed his own model, which combines crack pinning and crack bridging. This model is able to explain both the non-linearity and the maxima of Lange and Radford's data. Thus, it appears that these two types of toughening mechanisms, bridging and pinning, are interrelated, and it may be difficult to separate their contributions to the overall toughness.

Evidence for crack pinning in thermoplastic-modified epoxies has been presented by several investigators ${ }^{26,27}$. Again, the majority of these works show SEM micrographs in which 'tails' near the particles on the fracture surface could be observed. Kinloch et al. ${ }^{34}$ have published an optical micrograph that shows the crack front bowing between glass spheres in a glass-filled epxoy.

\section{Crack path deflection}

Another toughening mechanism often cited for producing the toughening effect in thermoplastic-modified epoxies is called crack path deflection. In this mechanism, the proposed role of the rigid thermoplastic particles is to cause the crack to deviate from its main plane. Such a deviation would result in increased surface area and would also reduce the mode I character of the opening of the crack (more mode II); either way, the energy required to propagate such a crack increases. Evidence for this mechanism is again found by observing the fracture surfaces with SEM. Quantitatively, the model ${ }^{35}$ may be stated as:

$$
\frac{K_{\mathrm{c}}}{K_{0}}=\left(\frac{E_{0}}{E_{\mathrm{c}}}\left(1+0.87 V_{\mathrm{f}}\right)\right)^{1 / 2}
$$

where $K_{\mathrm{c}}$ is the fracture toughness of the modified epoxy, $K_{0}$ is the fracture toughness of the unmodified epoxy, $E_{\mathrm{c}}$ is the Young's modulus of the modified epoxy, $E_{0}$ is the Young's modulus of the unmodified epoxy, and $V_{f}$ is the volume fraction of spheres.

Given that the ratio of Young's moduli is close to one for thermoplastic-modified epoxies and that the maximum volume fraction of thermoplastic modifier used is no greater than $30 \%$, then one may conclude that only modest increases would be observed when this mechanism is operating in thermoplastic modified epoxies: $K_{\mathrm{c}} / K_{0} \approx 1.12$.

\section{Toughening by particle-induced shear banding}

At least one group of researchers ${ }^{28}$ have argued that the toughening mechanism of thermoplastic-modified epoxies is similar to that of rubber particles - to induce shear banding in the matrix. In this case, the rigid plastic particles would yield, which lowers their modulus to that of a rubber; such a modulus mismatch would produce a significant stress concentration, which initiates shear banding in the matrix. The occurrence of particle-induced shear banding has been proposed as a result of a finite-element method (f.e.m.) model ${ }^{36}$ that considered the non-linear deformation of a rigid sphere embedded in a rigid matrix. Observations reported by Kim and Brown ${ }^{28}$ on thin films corroborate the occurrence of this mechanism in plane-strain situations.

\section{Summary}

Our understanding of the dominant toughening mechanisms for these thermoplastic-modified epoxies appears to be very incomplete. This situation makes it more difficult to choose the size, the chemical structure and the mechanical attributes of the second phase. Therefore, it is not surprising that in some cases the addition of a thermoplastic phase improves the toughness significantly while in other cases there is little change in toughness. It is surprising that more effort has not been applied to study the toughening mechanisms, since the thermoplastic particle approach is such an appealing method to toughen highly crosslinked epoxies. In addition to highly crosslinked systems, the use of thermoplastic particles to toughen 'ductile' epoxies is also appealing, since improved toughness may be achieved without lowering the modulus, yield stress, etc. 
The purpose of this investigation is to examine the toughening mechanisms in a newly developed thermoplastic-modified epoxy ${ }^{37}$ : an epoxy based on the diglycidyl ether of bisphenol A (DGEBA) cured with piperidine and modified with poly(phenylene oxide) (PPO). This particular modified epoxy was chosen since there is a multitude of work on rubber-modifying this particular epoxy matrix, which has been shown to be quite 'toughenable'. Serendipitously, the refractive index match between the epoxy resin and the PPO particles is so close that the cured plaques are transparent; this transparency facilitates the determination of the toughening mechanism when using optical microscopy. In addition to simple binary PPO/DGEBA blends, ternary blends containing a rubber modifier will also be examined.

\section{EXPERIMENTAL PROCEDURE}

\section{Materials}

All materials used in this investigation were from commercial sources and were used as received. The epoxy system consisted of a diglycidyl ether of bisphenol A based epoxy (epoxy equivalent weight $=186$ ) cured with piperidine. This epoxy system yields in tension, which provides the possibility that shear banding can be induced by a particulate phase. In this investigation, poly(phenylene oxide) (PPO; $M W=34000 \mathrm{~g} \mathrm{~mol}^{-1}$ ) was used to produce a particulate phase. The particulate morphology was obtained by first dissolving PPO in the hot epoxy and then precipitating spherical particles upon cure. The particulate morphology was stabilized by the addition of a styrene-maleic anhydride copolymer. Chemical structures of the starting materials can be found in Figure 2. The preparation of the PPO-epoxy blends is discussed in further detail in ref. 37. Compositional differences of the materials examined in this investigation are listed in Table 1.
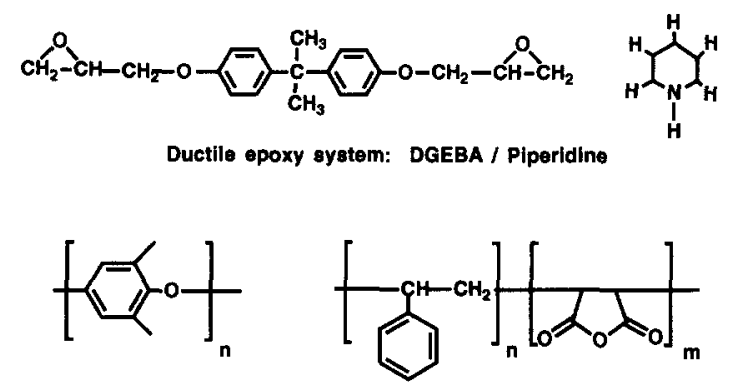

Rigid modifier: PPO

Copolymer: SMA

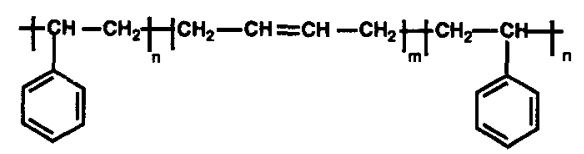

SBS Triblock copolymer

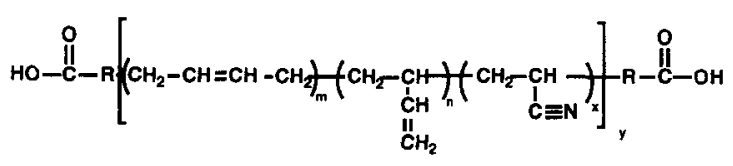

CTBN Elastomer

Figure 2 Chemical structures of organic materials used in this investigation

\section{Fracture toughness measurements}

Fracture toughness was measured by applying linear elastic fracture mechanics (l.e.f.m.). The critical planestrain stress intensity factor, $K_{\mathrm{lc}}$, was determined using a single-edge-notched (s.e.n.) specimen $(6.4 \mathrm{~mm} \times$ $12.7 \mathrm{~mm} \times 80 \mathrm{~mm}$ ) in a three-point bending geometry. These specimens were precracked by the following method: first, the sample was notched with a jeweller's saw; then, a razor blade, which had been chilled in liquid nitrogen, was inserted into the notch; next, the razor blade was struck by a rubber mallet to propagate a sharp crack well away from the notch; finally, the razor blade was quickly removed in order to reduce the size of the plastic zone at the crack tip. This method for precracking provided sharp cracks with negligible plastic deformation at the crack tip. The cracked specimen was then placed in a servohydraulic materials testing machine and loaded to fracture at a piston velocity of $2.54 \mathrm{~mm} \mathrm{~s}^{-1}$. The reported $K_{\text {Ic }}$ values are the average of a minimum of six tests.

The critical stress intensity factors were calculated using the following relations ${ }^{38}$ :

$$
K_{\mathrm{Ic}}=Y \frac{3 P S \sqrt{ } a}{2 t w^{2}}
$$

where $P$ is the critical load for crack propagation, $S$ is the length of the span, $a$ is the crack length, $t$ is the thickness, $w$ is the width, and $Y$ is the non-dimensional shape factor, given by:

$$
Y=1.9-3.07(a / w)+14.53(a / w)^{2}-25.11(a / w)^{3}+25.80(a / w)^{4}
$$

The effect of crack-tip plasticity on the validity of the $K$ measurements was evaluated using the ASTM E399 standard equation, which determines whether the thickness is large enough to provide the plane-strain constraint: $t>2.5\left(K_{\mathrm{Ic}} / \sigma_{\mathrm{y}}\right)^{2}$. All specimens evaluated in this work have the requisite dimensions for plane-strain conditions.

\section{Uniaxial tensile dilatometry}

Tensile dilatometry was employed to study micromechanical deformation mechanisms that occur in tension. The technique used in this investigation was modelled after that used by Bucknall ${ }^{39}$, except that a constant displacement rate was used rather than a constant load. Details of our tensile dilatometry technique are given elsewhere ${ }^{40}$. An ASTM D638 standard stroke rate of $50 \mathrm{~mm} \mathrm{~min} \mathrm{~min}^{-1}$ is used. Three specimens for each formulation were examined. The volume strain is plotted up to the point of necking, since the strain response is no longer homogeneous after neck formation. Young's modulus is obtained from a linear least-squares fit of the stress-strain data taken from 0.1 to $0.5 \%$ strain. The maximum in the engineering stress is reported here as the yield stress.

\section{Fractography}

Fractographic methods such as SEM and transmission optical microscopy (TOM) were employed to elucidate the toughening mechanisms.

SEM is used to examine the fracture surfaces of three-point bend and uniaxial tensile specimens. These examinations are performed using a Hitachi S800 scanning electron microscope in the conventional 
Toughening in thermoplastic-modified epoxies. 1: R. A. Pearson and A. F. Yee

Table 1 List of formulations: (a) series 1, the effect of poly(phenylene oxide) (PPO) content; (b) series 2, the influence of rubber addition

\begin{tabular}{|c|c|c|c|c|c|}
\hline Designation $^{a}$ & $\begin{array}{l}\text { DGEBA } \\
\text { (g) }\end{array}$ & $\begin{array}{l}\text { SMA } \\
\text { (g) }\end{array}$ & $\begin{array}{l}\text { Piperidine } \\
\text { (ml) }\end{array}$ & $\begin{array}{l}\text { PPO } \\
(\mathrm{g})\end{array}$ & $\begin{array}{l}\text { Rubber } \\
\text { (g) }\end{array}$ \\
\hline \multicolumn{6}{|l|}{ (a) Series 1} \\
\hline DGEBA/PIP & 500 & - & 29 & - & - \\
\hline DGEBA/SMA/PIP & 500 & 12.5 & 29 & - & - \\
\hline DGEBA/SMA/PIP/PPO(5) & 500 & 12.5 & 29 & 25 & - \\
\hline DGEBA/SMA/PIP/PPO(10) & 500 & 12.5 & 29 & 50 & - \\
\hline DGEBA/SMA/PIP/PPO(15) & 500 & 12.5 & 29 & 75 & - \\
\hline DGEBA/SMA/PIP/PPO $(\sim 20)$ & 500 & 12.5 & 29 & $\sim 100$ & - \\
\hline \multicolumn{6}{|l|}{ (b) Series 2} \\
\hline DGEBA/SMA/PIP/PPO(10) & 500 & 12.5 & 29 & 50 & - \\
\hline DGEBA/SMA/PIP/PPO(10)/SBS(2.5) & 500 & 12.5 & 29 & 50 & $12.5^{b}$ \\
\hline DGEBA/SMA/PIP/PPO(10)/CTBN(5) & 500 & 12.5 & 29 & 50 & $25^{c}$ \\
\hline DGEBA/SMA/PIP/CTBN(5) & 500 & 12.5 & 29 & - & $25^{c}$ \\
\hline
\end{tabular}

${ }^{a}$ DGEBA, diglycidyl ether of bisphenol A; PIP, polyisoprene; SMA, styrene-maleic anhydride; SBS, styrene-butadiene-styrene; CTBN, carboxyl-terminated butadiene-acrylonitrile

${ }^{b}$ Denotes Kraton D1102 resin used

${ }^{\circ}$ Denotes Hycar CTBN 1300X8 resin used

secondary electron imaging technique mode. The accelerating voltage was $10 \mathrm{kV}$. Samples are prepared by sputtering a thin layer of gold-palladium on the fracture surface in order to reduce any charge build-up (charging).

TOM is used to examine damage zones at the crack tips of four-point bend specimens as well the permanent deformation occurring in uniaxial tensile specimens. These examinations are performed using a Nikon Microphot II optical microscope. A conventional tungsten light source is used. The microscope is equipped with crossed polars, which are used to detect plastic deformation. The sample preparation is rather involved: (1) a double-notched four-point bend (d.n.-4p.b.) method ${ }^{41}$ is used to obtain mature damage zones; (2) the damage zones are removed (sectioned) with a diamond saw and placed in a mounting epoxy; and (3) thin sections are obtained by petrographically polishing sections to their mid-planes.

\section{RESULTS AND DISCUSSION}

\section{Morphology}

Morphological features of the various PPO-modified epoxies can be found in the SEM micrographs contained in Figure 3. Such micrographs show a particulate morphology for PPO-modified epoxies containing 5 and $10 \mathrm{phr}$ PPO, and also show the co-continuous nature of PPO-modified epoxies containing larger amounts of PPO. Similar phase behaviour in other thermoplasticmodified epoxies has been reported in the literature ${ }^{23,24}$. Unique to our study are (1) the use of a copolymer to stabilize the particulate morphology and (2) the investigation of ternary blends of PPO/SBS (styrenebutadiene-styrene) and PPO/CTBN (carboxyl-terminated butadiene-acrylonitrile) with epoxy. The PPO/SBS combination leads to irregularly shaped particles containing glassy inclusions - very similar to classic high-impact polystyrene (HIPS) particles.

\section{Fracture toughness}

The fracture toughness of PPO-modified epoxies increases almost linearly with PPO content. These results are presented graphically in Figure 4 and are summarized in Table 2. The simple linear relation between PPO content and fracture toughness is surprising since
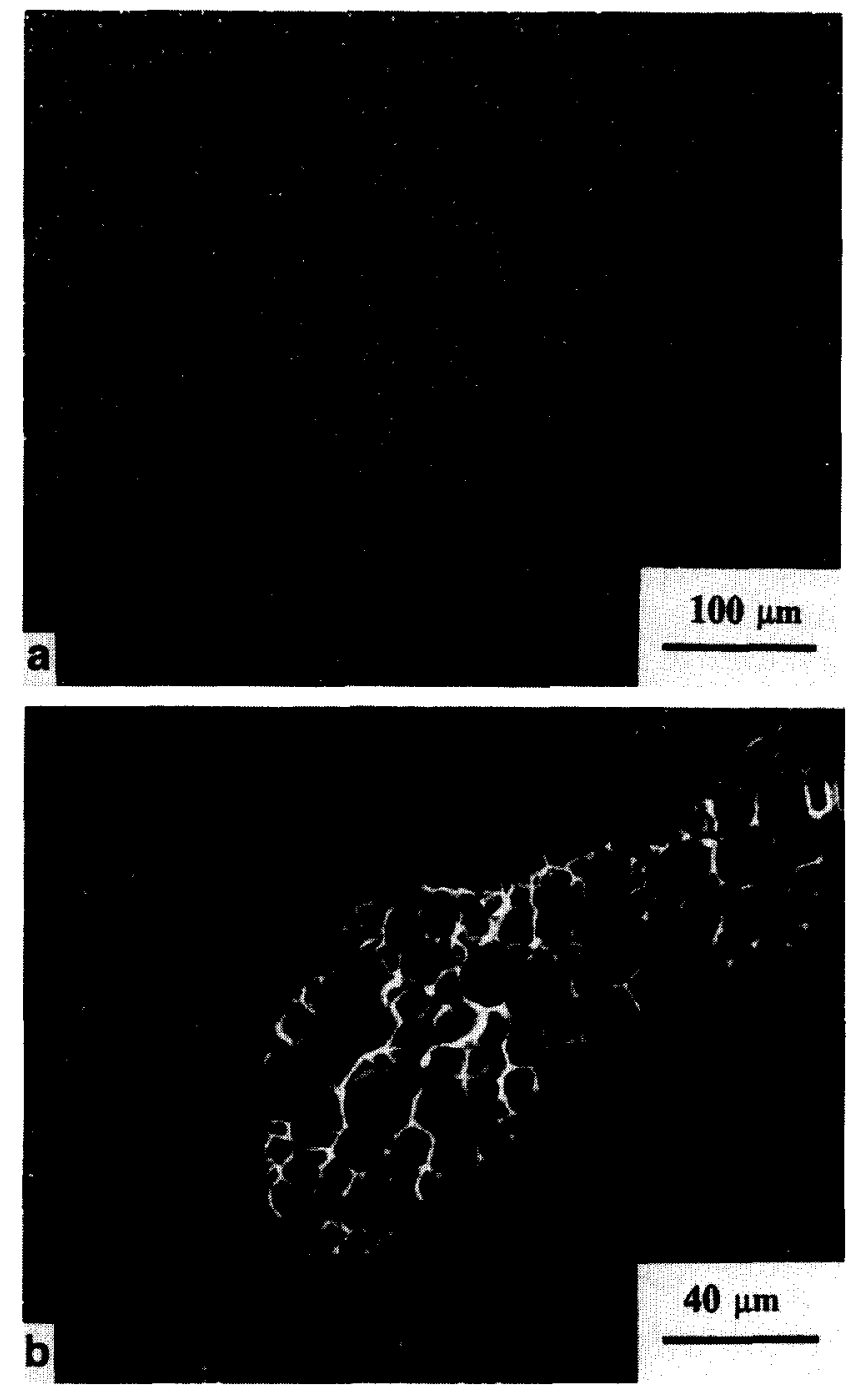

Figure 3 SEM micrographs illustrating: (a) uniform dispersion of PPO particles and (b) small, homogeneous PPO particles plus large co-continuous PPO-epoxy particles

the morphology undergoes a dramatic change from a particulate morphology to one that consists of cocontinuous domains. A similar linear increase in fracture toughness has been reported in the literature for other thermoplastic-modified epoxies ${ }^{24,27}$. 
Table 2 Summary of the mechanical behaviour of PPO-modified epoxies: (a) series 1, the effect of PPO content; (b) series 2, the influence of rubber addition

\begin{tabular}{llll}
\hline Designation & $\begin{array}{l}\text { Emodulus } \\
\text { (GPa) }\end{array}$ & $\begin{array}{l}\text { Yield stress } \\
(\mathrm{MPa})\end{array}$ & $\begin{array}{l}K_{\mathrm{lc}} \\
\left(\mathrm{MPa} \mathrm{m} \mathbf{m}^{1 / 2}\right)\end{array}$ \\
\hline (a) Series 1 & & & 1.1 \\
DGEBA/PIP $^{a}$ & 3.1 & 79 & 1.2 \\
DGEBA/SMA/PIP/PPO(5) & 3.1 & 80 & 1.4 \\
DGEBA/SMA/PIP/PPO(10) & 3.2 & 80 & 1.6 \\
DGEBA/SMA/PIP/PPO(15) & 3.2 & 89 & 1.8 \\
DGEBA/SMA/PIP/PPO( 20) & 3.0 & & 1.5 \\
(b) Series 2 & & 79 & 1.9 \\
DGEBA/SMA/PIP/PPO(10) & 3.0 & 71 & 2.1 \\
DGEBA/SMA/PIP/PPO(10)/SBS(2.5) & 3.0 & 74 & 2.1 \\
DGEBA/SMA/PIP/PPO(10)/CTBN(5) & 2.9 & - & \\
DGEBA/SMA/PIP/CTBN(5) & - & & \\
\hline
\end{tabular}

"Note that DER 332 resin was used

${ }^{b}$ Note that DER 331 resin was used

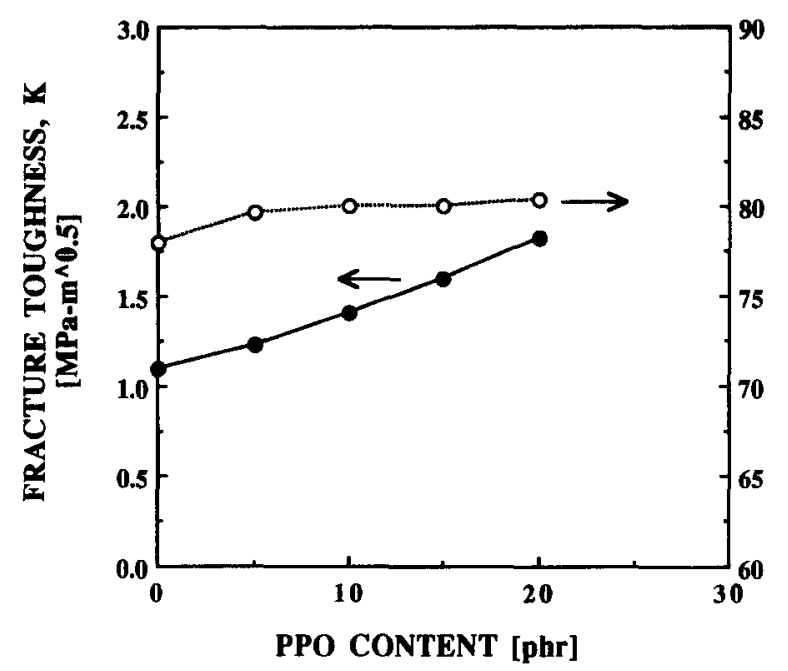

Figure 4 Fracture toughness increases with PPO content while the yield strength remains constant

The fracture toughness results for the ternary blends are also shown in Table 2. Two striking results are obtained. First, a small amount of SBS rubber results in a dramatic increase in fracture toughness in PPOmodified epoxies. It was our intention to add a rubbery phase to the particle to facilitate cavitation, which would promote shear banding. It will be shown in the section on fractography that this scenario is not correct. The second striking feature is that, although the addition of CTBN rubber improves the toughness of PPO-modified blends, the effect is no greater than if the epoxy was modified with CTBN alone, i.e. the interaction between the two types of toughening mechanisms is not additive and it appears that they might even be competing.

\section{Tensile dilatometry}

Tensile dilatometry was applied to determine the toughening mechanisms in PPO-modified epoxies. Figure 5 suggests that the micromechanical deformation mechanism operating in uniaxial tension involves shear deformation and is very similar to that of the unmodified epoxy. Examination of petrographically polished thin sections corroborate this state (see next subsection). However, it will be shown that the examination of the damage zones in front of crack tips of PPO-modified epoxies leads us to conclude that a different micromechanical

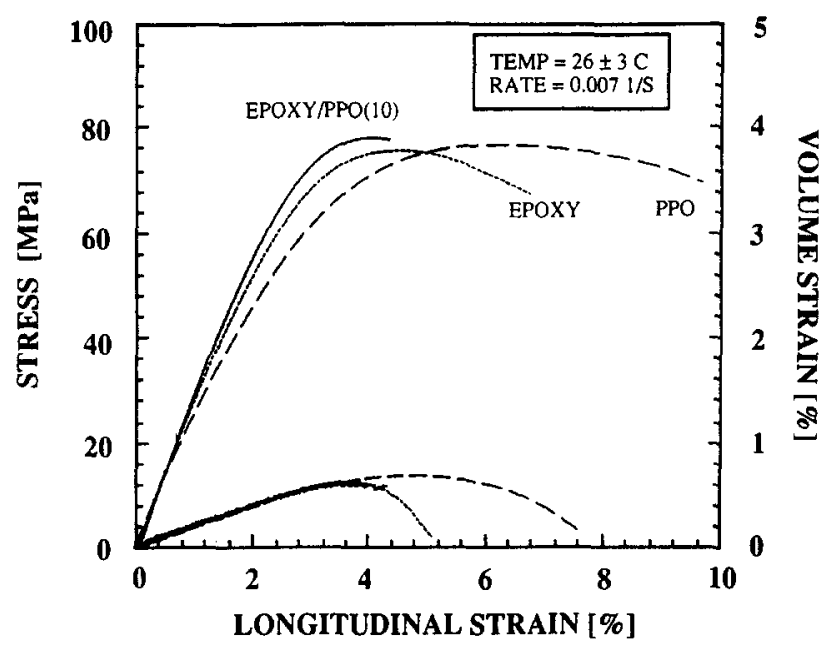

Figure 5 Tensile behaviour of DGEBA epoxy, PPO resin and PPO-modified epoxy

deformation mechanism dominates at the crack tip. This result identifies an inherent weakness of the tensile dilatometric method; tensile dilatometry assumes that the micromechanical deformation mechanisms are not stressstate-dependent, i.e. the same mechanisms operating in simple uniaxial tension are also operating at the crack tip, which has a much greater triaxial component. The difference in strain rates may also be a contributing factor.

Tensile dilatometry of ternary blends also indicated that no additional dilative mechanism was occurring prior to yield. Once again, this suggests shear banding to be the major deformation mechanism. Also, once again, our microscopic examinations corroborate the tensile dilatometric results when performed on tensile specimens. However, different mechanisms are observed when d.n.-4p.b. specimens are examined. The microscopic investigations are discussed next.

\section{Fractography}

Three different specimens were analysed: uniaxial tensile, double-notched four-point bend, and doublecracked four-point bend specimens. The triaxial nature of the stress field increases respectively.

The nature of the micromechanical deformation mechanisms of DGEBA/PIP/PPO alloys subjected to 
uniaxial tension can be best explained by examining Figure 6. Figure 6 is an optical micrograph of a thin section taken from the mid-section near the necked region of a uniaxial tensile bar of DGEBA/PIP/PPO(10). This section is viewed using cross-polarized light, which facilitates the observation of shear bands. Indeed, the shear bands observed are identical in nature to those triggered by rubber particles in similar rubber-modified materials. Interestingly, even with the small modulus mismatch between PPO and DGEBA/PIP, the PPO particles are able to initiate shear bands in this epoxy. For a more detailed discussion as to the formation of shear bands, see ref. 41.

The evidence presented thus far suggests that the toughening mechanism for PPO-modified epoxy might consist of massive shear banding; however, this is not the case. Figure 7 is an example of the type of damage zone seen at the crack tip of a PPO-modified epoxy: the crack bifurcates under load and no evidence of a birefringent plastic zone is found. Thus, we contend that multiple crack bifurcation, a form of microcracking, contributes to the overall toughness. The multiple crack bifurcations occur due to the presence of the PPO particles, without
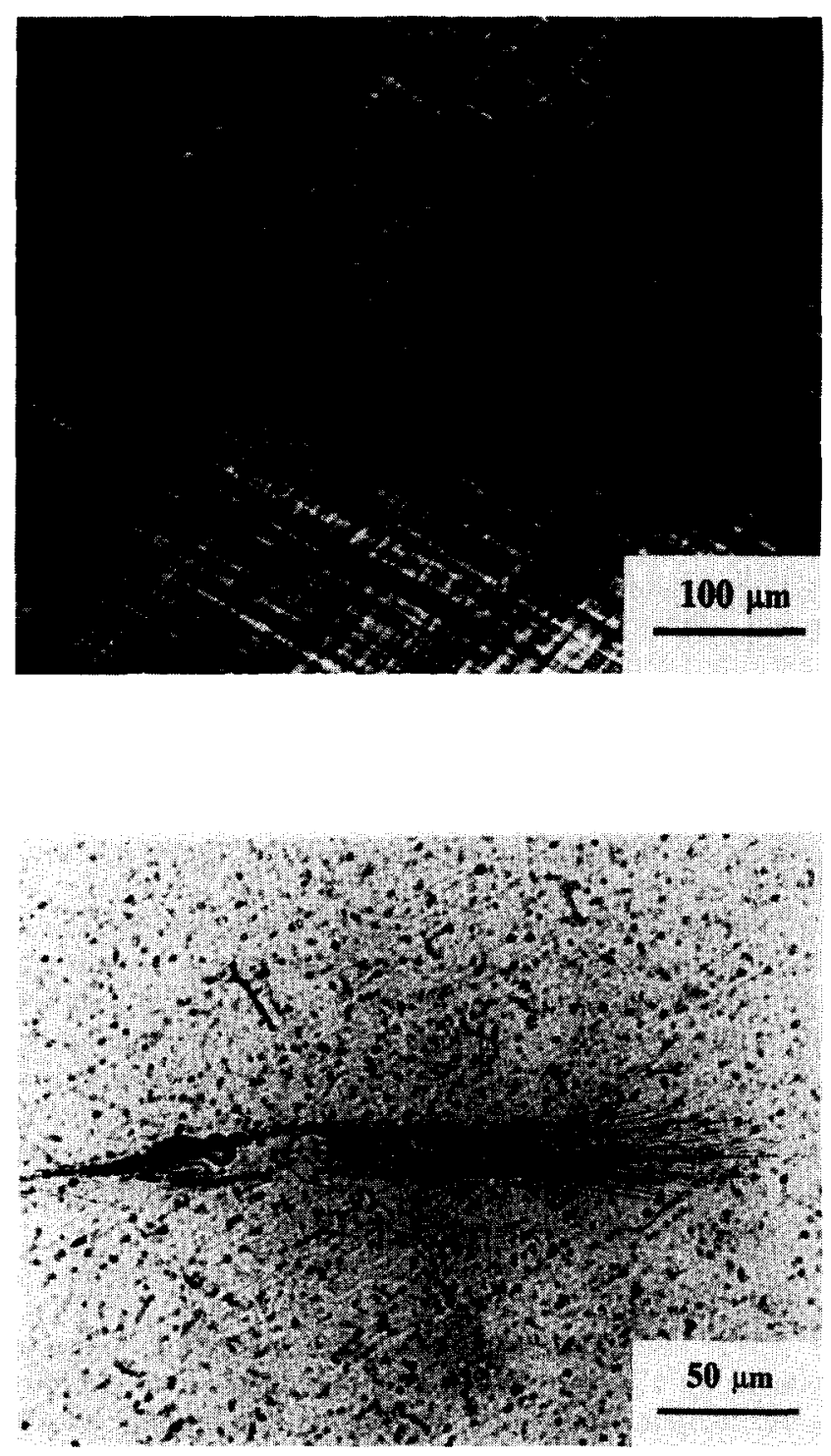

Figure 7 Optical micrograph of a thin section taken from an s.e.n. specimen of a PPO-modified epoxy. Note the presence of crack branching and microcracks which this effect does not occur. In addition to crack bifurcation, SEM examinations of the fracture surfaces at these bifurcation points indicate that the PPO particles are being torn. Thus, crack bridging by these ductile PPO particles also contributes to the overall toughness; however, the amount of elongation and tearing appears negligible. Therefore, we assert that multiple crack bifurcation, not crack bridging, is the major toughening mechanism. Although crack bridging is not the major toughening mechanism, it is thought to play a crucial role in facilitating the size of the damage zone. This will be explained below.

The source of the toughness improvements in PPOmodified epoxies can be determined by examining Figure 8. Figure 8 contains SEM micrographs of the fracture surface in the slow growth region of the s.e.n. specimens. These micrographs show some signs of particle tearing. Features of Figure $8 d$ indicate that phase inversion may have occurred. Similar features have been shown for an epoxy modified with $30 \mathrm{phr}$ PSF by Hedrick et al. ${ }^{23}$, who attributed the source of toughness improvement solely to particle bridging. Despite the similarities of the fracture surfaces, the optical micrographs of the subsurface damage of the PPOmodified epoxies show the presence of microcracks.

The presence of microcracks in PPO-modified epoxies is established by examining the subsurface damage zones in front of notch tips and at crack tips. The damage zones in front of a V-notch were obtained using a d.n.-4p.b. specimen and these zones are shown in Figure 9. Microcracks can be seen near the elastic-plastic interface, which corresponds to the region of maximum hydrostatic tensile stress. The microcracks grow outwards towards the elastically deformed material, and very little growth is observed in the plastic zone, which may indicate that the triaxial tension is higher in the forward direction. Microcracks are also observed at the crack tips of PPO-modified epoxy. Figure 10 contains OM micrographs of the damage zone at the crack tips of DER 332/PIP/PPO(10)/SMA(2.5) and DER 332/PIP/ PPO( 20)/SMA(2.5). All of the microcracks appear to have initiated from one point, i.e. the main crack bifurcates. It is important to note that the size of this microcracking zone correlates with the improvement in toughness.

Although we were successful in producing thermoplastictoughened epoxies, the amount of toughness improvement was quite small. The amount of toughness is reasonable when one considers the size of the damage zone and the fact that the shear banding mechanism is not operating at the crack tip of these materials. In an earlier study, the potency of the shear banding mechanism was illustrated ${ }^{21}$. To facilitate the occurrence of shear banding, small amounts of rubber were added to DGEBA/PIP/PPO blends. Two types of rubbers were examined: SBS, which should reside in the PPO phase, and CTBN, which should reside in the epoxy matrix as a separate phase. As mentioned previously, the addition of each type of elastomer increased toughness. Let us now elucidate the toughening mechanisms operating in these ternary blends.

The source of the toughness improvements for the DGEBA/PIP/PPO(10)/CTBN(5) material can be determined by examining the fracture surface and the subsurface damage using SEM and OM respectively. Figure 11 contains an SEM micrograph taken in the 

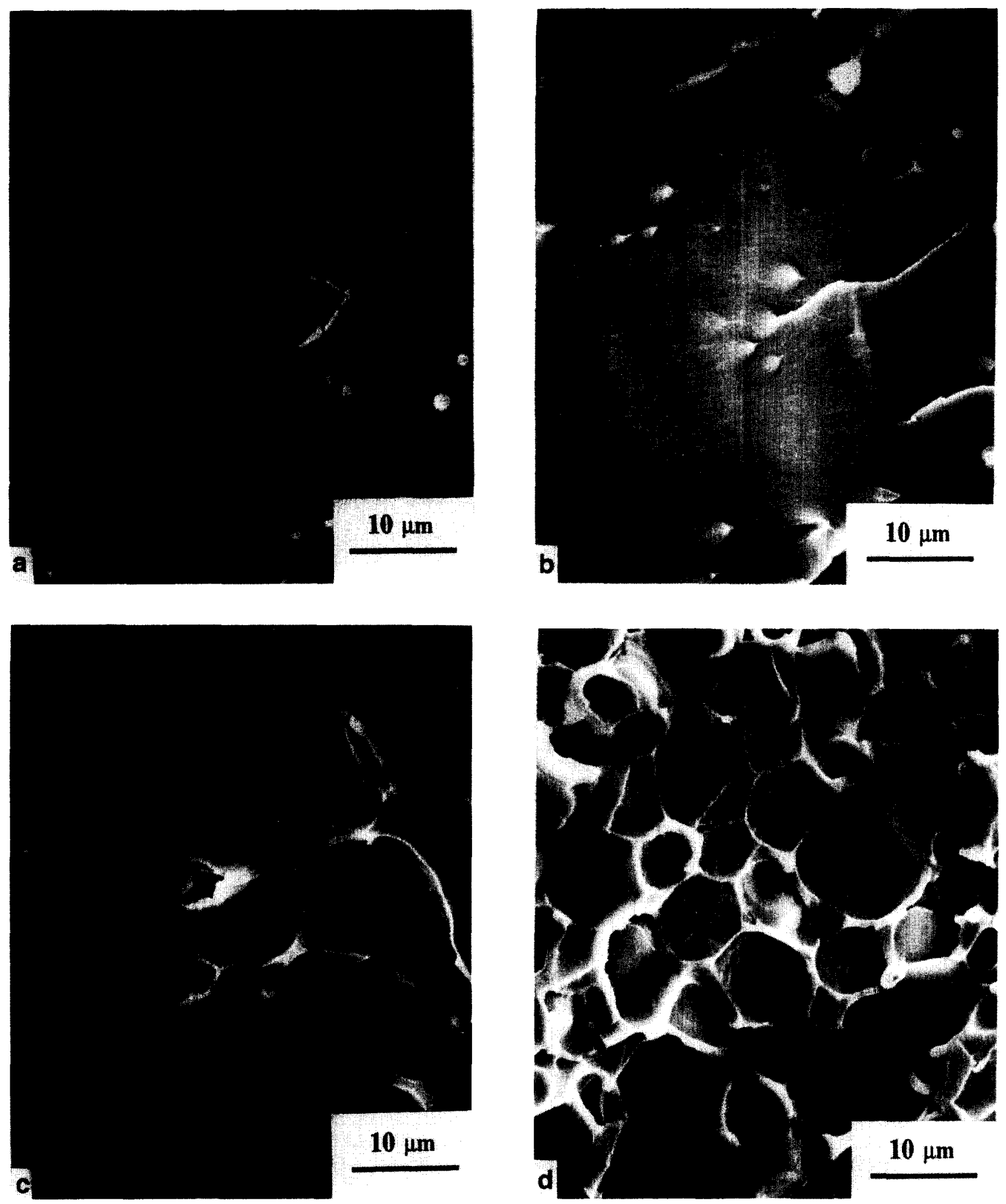

Figure 8 Scanning electron micrographs of the s.e.n. fracture surfaces of: (a) DGEBA/PIP/PPO(5), (b) DGEBA/PIP/PPO(10), (c) DGEBA/PIP/PPO(15), and (d) DGEBA/PIP/PPO(20). Note the presence of torn PPO particles

stress-whitened region; the micrograph contains the same features found in a CTBN-modified epoxy. A similar observation is true when the subsurface damage zone is observed in OM (see Figure 11). Combining these observations with the fracture toughness results leads to the conclusion that the bifurcation mechanism is suppressed by the cavitating particles. One can rationalize this effect since rubber particles are added to relieve triaxial stress and we surmise that it is the triaxial stress that promotes microcracking. From these results, one can also surmise that the PPO particles can still act as bridging particles and it is important to note that this mechanism does not increase the toughness of the DGEBA/PIP/PPO(10)/CTBN(5) material more than the CTBN-modified epoxy.

The source of toughness improvement for the DGEBA/ 

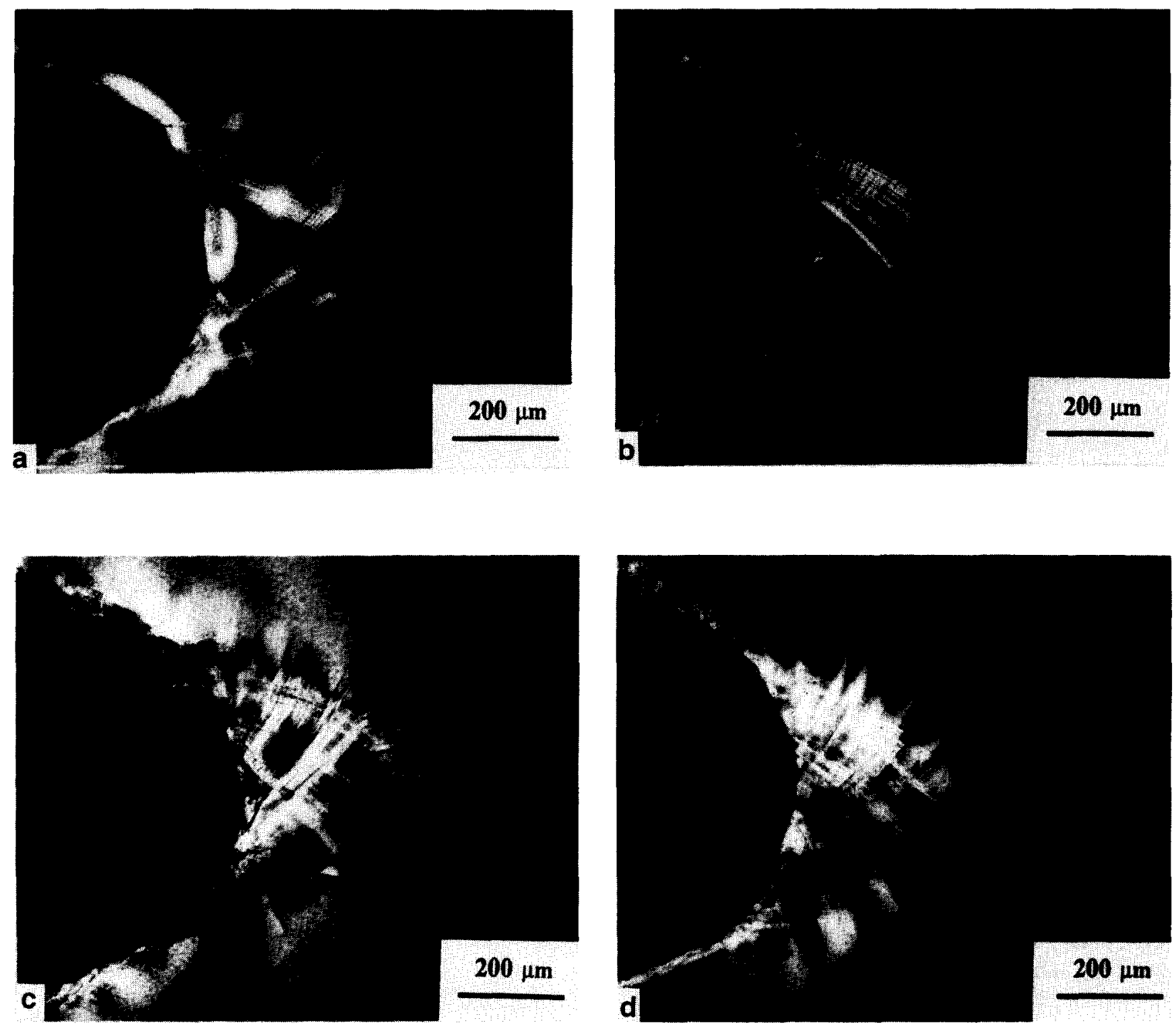

Figure 9 Optical micrographs of the damage zone of the V-notched s.e.n. specimens show signs of microcracking at the elastic-plastic boundary. These micrographs were taken using crossed polars and a quarter-wave plate

PIP/PPO(10)/SBS(2.5) material is determined by examining the fracture surface and the subsurface. Figure 12 contains an SEM micrograph taken in the stress-whitened region; in contrast to the previous ternary blend containing CTBN, the DGEBA/PIP/PPO(10)/SBS(2.5) material exhibits a unique fracture surface containing many 'welts and tuffs'; these observations suggest the presence of many secondary cracks interacting with the main crack. The OM micrograph in Figure $13 b$ corroborates the occurrence of microcracks at the crack tip of this material; the microcracks appear to be induced by the composite PPO/SBS particles. Interestingly, the same particles induce shear banding in the epoxy matrix when tested in uniaxial tension (see Figure 14).

\section{DISCUSSION}

\section{Microcracking mechanisms}

Several models have been developed to estimate the contribution of microcracking to the overall fracture toughness ${ }^{42-45}$. In addition to the many models, several types of microcracking mechanisms have been identified.
Three types of microcracking mechanisms in two-phase materials are schematically drawn in Figure 15. For the materials investigated in this work, the initiation of microcracks in the PPO particles appears to be a logical choice. However, in addition to microcracking, the main crack often exhibits branching. The effect of branching and microcracking on the fracture toughness of ceramic materials has been discussed recently by Gao and Wang $^{45}$. Figures $16-18$ illustrate their analysis. Mathematically speaking, the increase in toughness is related to the angle of the main crack and the density of the microcracks through the following equation:

$$
\begin{aligned}
\frac{K_{\mathrm{c}}}{K_{0}} & =\frac{1}{1-(\pi / 2)[\rho /(3-\pi \rho)]^{1 / 2}} \\
& \times\left[[2 \cos (\beta / 2)]^{\log 2 / \log [2 \cos (\beta / 2)]-1}-\frac{\rho}{a}\left(\frac{\rho}{1-\pi \rho / 2}\right)\right]^{1 / 2}
\end{aligned}
$$

where $K_{\mathrm{c}}$ is the fracture toughness of a material containing microcracks, $K_{0}$ is the fracture toughness of 

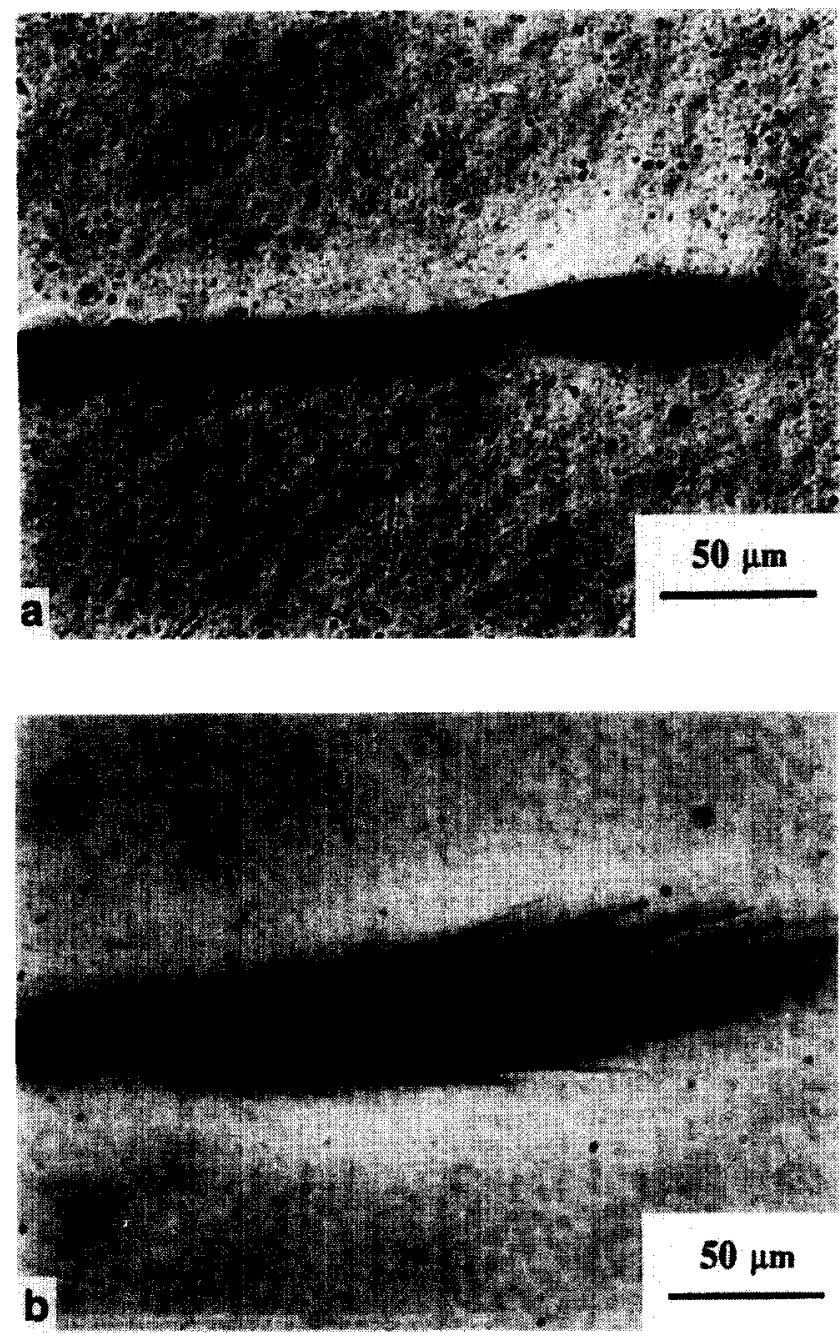

Figure 10 Optical micrographs of the damage zone of the cracked s.e.n. specimens show signs of bifurcating microcracks. The size of the microcracked zone is a function of PPO content: (a) DGEBA/PIP/PPO(10) and (b) DGEBA/PIP/PPO $(\sim 20)$

a material without microcracks, $\beta$ is angle of the branched crack, and $\rho$ is the density of microcracks.

A plot of $K_{\mathrm{c}} / K_{0}$ versus branching angle for various microcracking densities is very illustrative (see Figure 17): the interaction between branching and microcracking is rather large. Parenthetically speaking, one must remember that this is a model developed from limited experimental results on one specific type of ceramic material; the universality of the model has yet to be proven. Qualitatively, the model appears to be appropriate as a starting point for the PPO-modified epoxies investigated in this work. The material parameters used to generate Figure 18 appear to be reasonable.

The model proposed by Gao and Wang can assist in analysing the fracture toughness results for the PPOmodified epoxies. Part of the increase in toughness is due to the bifurcation of the crack tip, which acts as a shielding mechanism. Further crack shielding occurs by increasing the density of microcracks, which is achieved by adding more PPO particles. Modifying the PPO particles with SBS increases the number of microcracks per particle, which in turn increases the microcrack density, hence toughness. Presumably, the role of the SBS in the PPO particles is to initiate crazes in the PPO phase, which
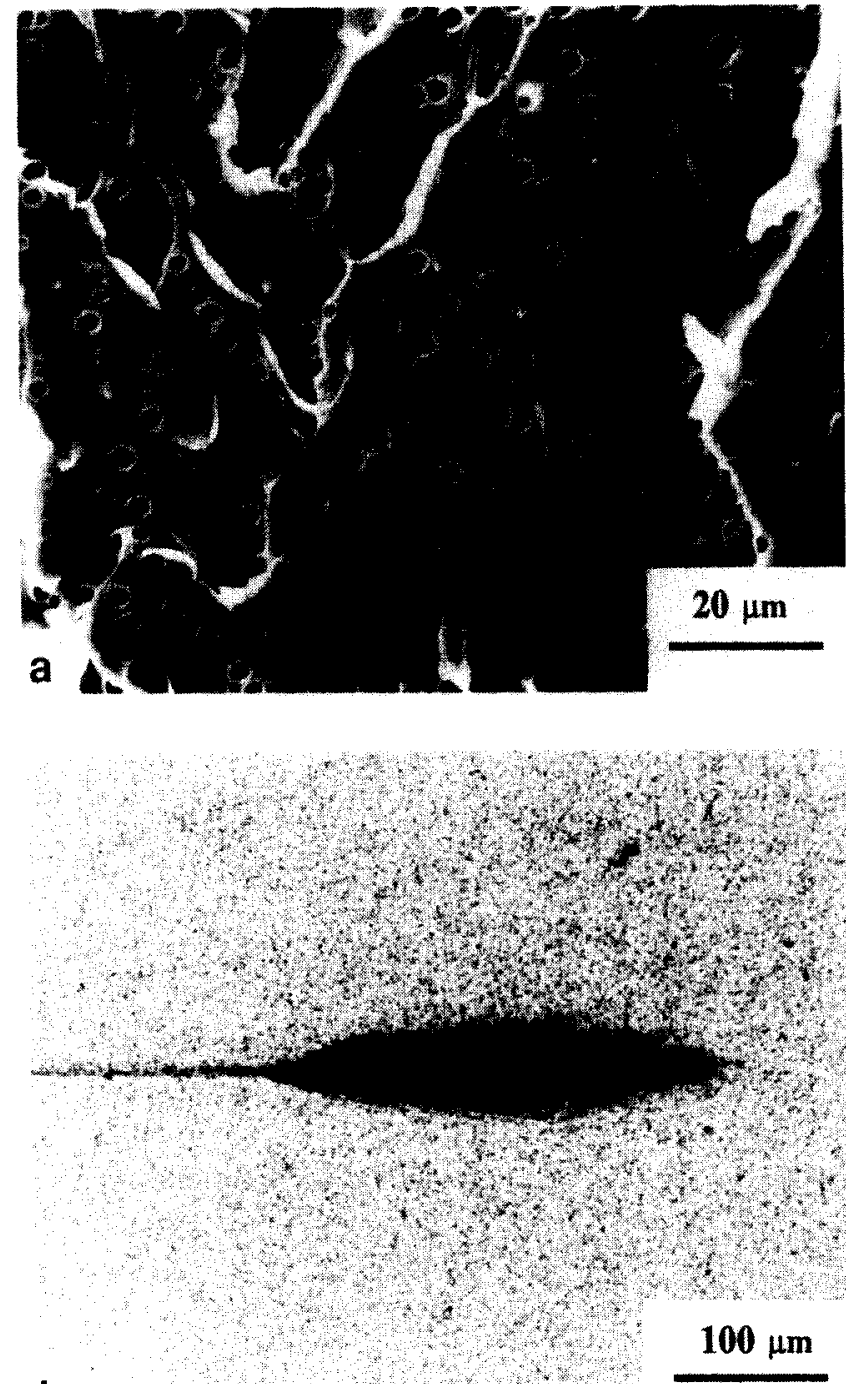

b

Figure 11 Fractography of ternary alloy containing DGEBA/PIP/ PPO(10)/CTBN(5): (a) scanning electron micrograph shows dilated CTBN particles and torn PPO particles, and (b) optical micrograph shows rubber particle cavitation but no evidence of microcracking

propagate into the epoxy matrix where they are transformed into microcracks. Crazes within similar particles have been reported for a PPO/PA (polyamide) alloy $^{46}$

Particle bridging is often reported as the toughening mechanism for thermoplastic-modified thermosets; this conclusion has been made based solely on the examination of fracture surfaces using SEM. We have reported similar micrographs that show ductile tearing of the thermoplastic particles. However, we have shown that the crack is far from planar, as one would assume if only a bridging mechanism was operating. Moreover, we have shown that by increasing the microcrack density one can further improve toughness. Therefore, future research should focus on those parameters which control the density of microcracks; investigating the role of interfacial properties seems like a likely avenue to explore. In fairness to the particle bridging proponents, particle bridging could be very important in stabilizing the microcracks, and hence important in controlling the size of the microcracking zone.

Another important implication of this work is that, since shear plasticity did not play an important role in 


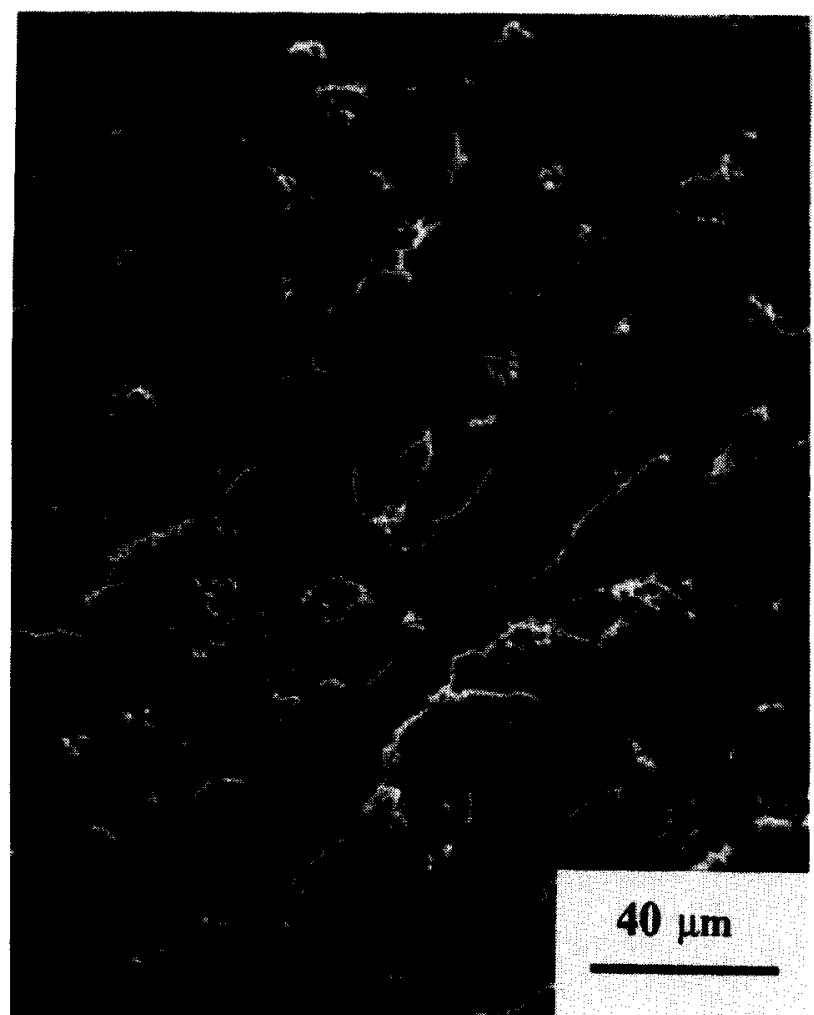

Figure 12 Scanning electron micrograph reveals many fracture steps on the surface of an s.e.n. specimen comprised DGEBA/PIP/PPO(10)/ SBS(2.5)
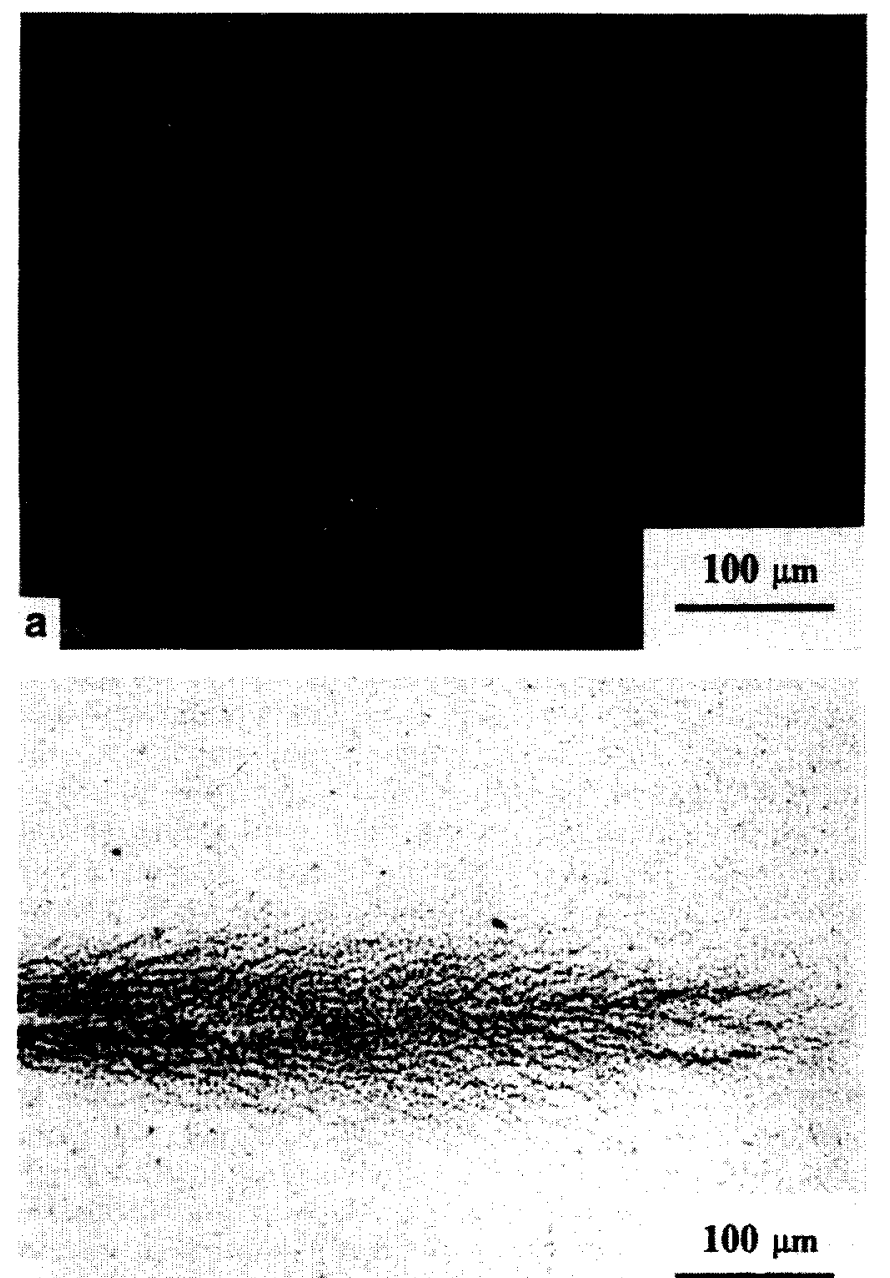

C

Figure 13 Optical micrographs illustrating the lack of shear yield in (a, b) DGEBA/PIP/PPO(10)/SBS(2.5) as compared to (c, d) a rubber-modified epoxy DGEBA/PIP/CTBN(10); bright field $(a, c)$ and crossed polars $(b, d)$

toughening, the mechanisms here may be effective in toughening much more brittle epoxies than that used in this work. Indeed, observations by Kim and Robertson on a poly(butylene terephthalate)-toughened brittle epoxy ${ }^{47}$ can be consistently explained by the mechanisms proposed here.

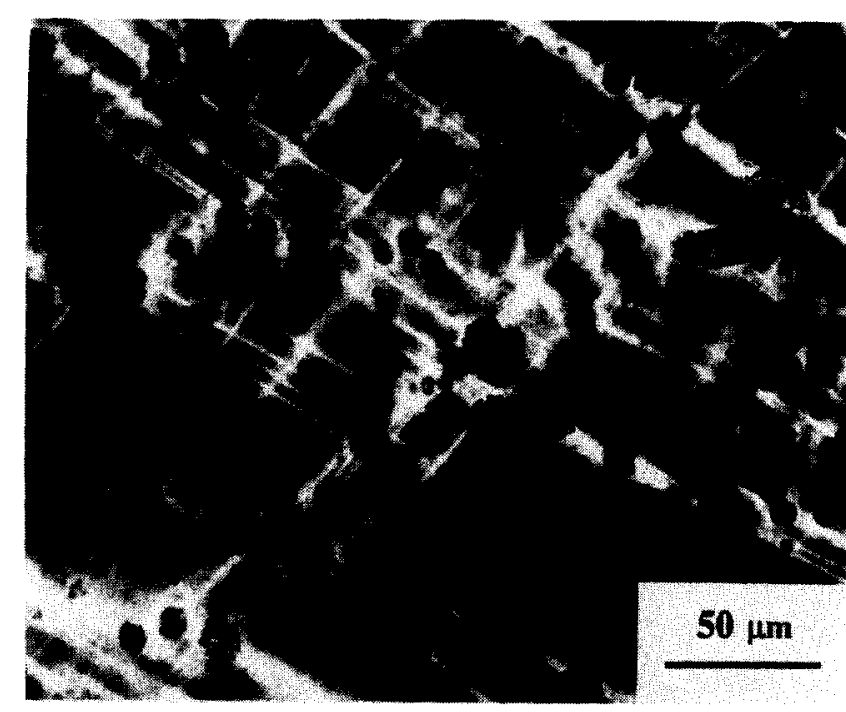

Figure 14 An optical micrograph of a thin section taken from a tensile specimen of DGEBA/PIP/PPO(10)/SBS(2.5). Note the presence of shear bands
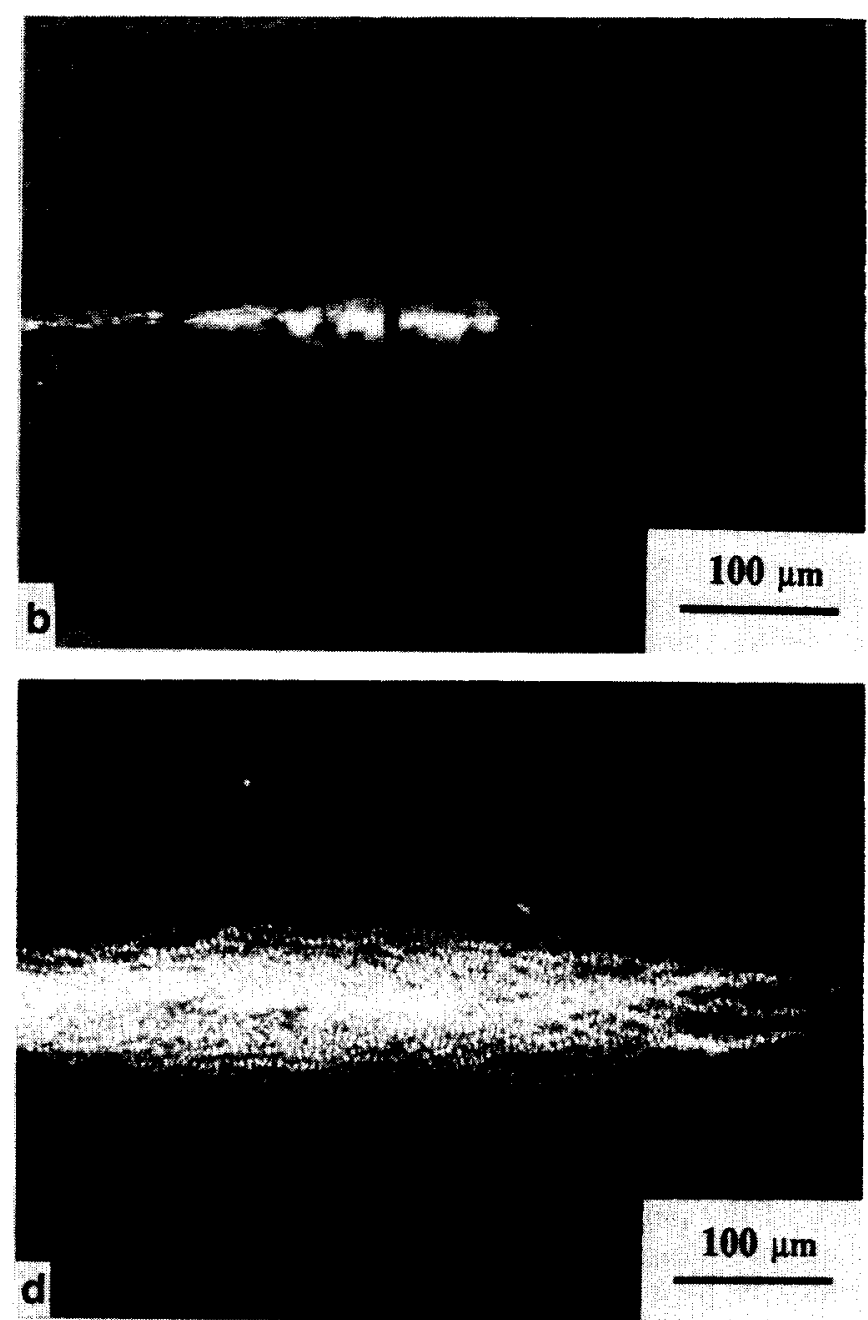


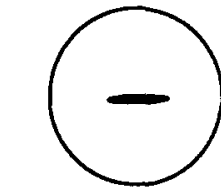

a

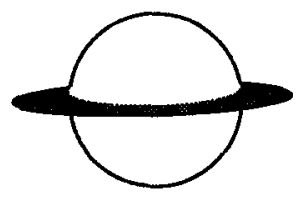

b

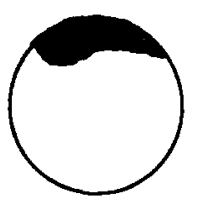

C

Figure 15 Three possible types of microcracks: (a) particulate, (b) matrix and (c) interfacial

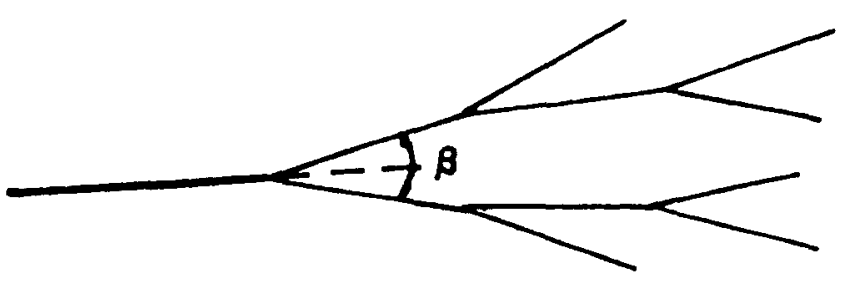

a

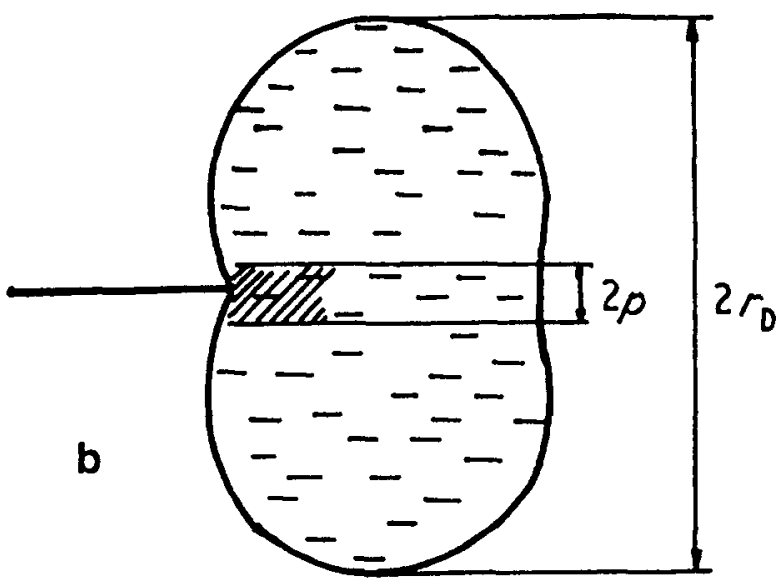

Figure 16 Schematic diagrams of microcracking model proposed by Gao and Wang ${ }^{45}$ : (a) the model of the branching of the crack; (b) main crack tip showing 'dissipation zone' and process zone

\section{SUMMARY AND CONCLUSIONS}

In summary the following observations and conclusions have been made:

A ductile epoxy has been modified with thermoplastic resin, which resulted in spherical, $2 \mu \mathrm{m}$ PPO particles embedded in a ductile epoxy matrix.

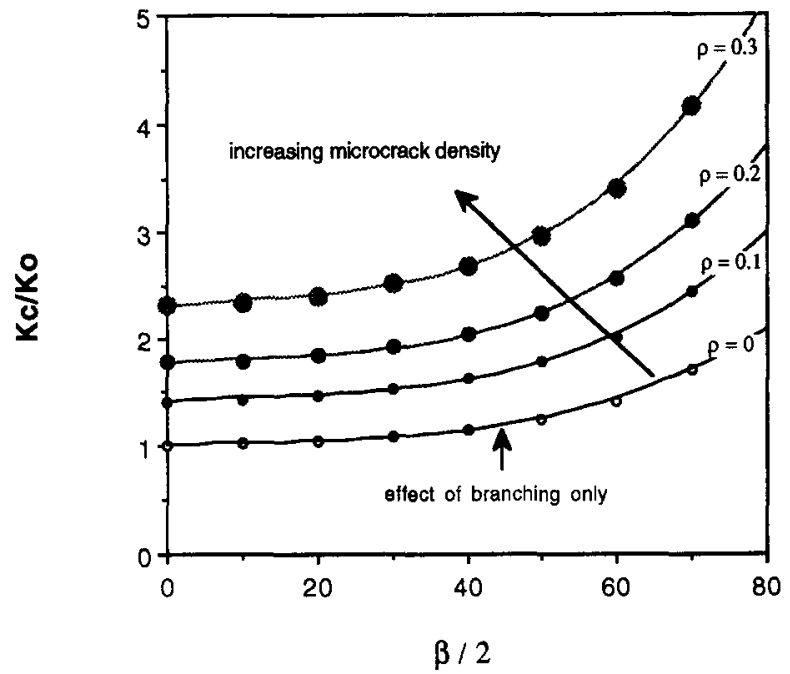

Figure 17 The model proposed by Gao and Wang ${ }^{45}$ emphasizes the benefits of increasing both the density of microcracks and the branching angle on fracture toughness.

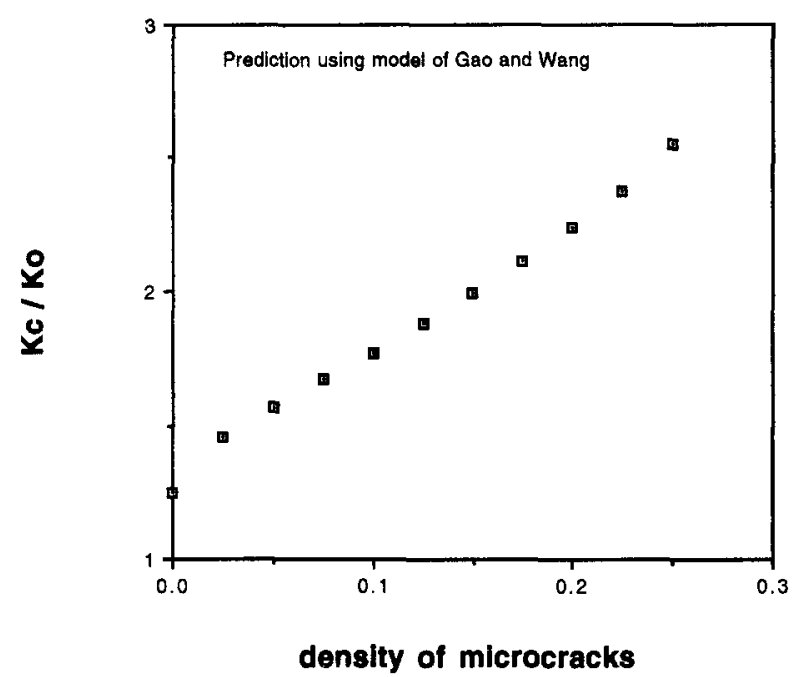

Figure 18 The model proposed by Gao and Wang ${ }^{45}$ predicts that the fracture toughness should increase in a linear fashion as the microcrack density increases (in their prediction shown, $\beta / 2=50^{\circ}$ and $\rho / a=0.25$ )

The PPO particles are able to initiate shear bands in the epoxy matrix in uniaxial tension.

The addition of PPO improves the fracture toughness of this ductile epoxy; the amount of toughness improvement is almost a linear function of the volume fraction of PPO spheres. It is important to note that tensile yield strength does not increase with PPO content but remains constant.

The toughening mechanism is not massive shear banding as has been found for rubber-modified epoxies; instead, the PPO-modified epoxies are toughened by a microcracking mechanism.

The density of microcracks can be increased by adding SBS triblock copolymers, which exist in the PPO phase. These composite particles are able to initiate several mirocracks per particle.

In contrast, the addition of CTBN particles, which exist in the epoxy matrix, suppresses the microcracking mechanism and the cavitation/shear banding mechanisms dominate.

It is thought that if one is able to initiate microcracks in a highly crosslinked epoxy then the amount of 
toughness improvement would be greater than that obtained by rubber toughening since matrix plasticity is not required and the microcracking mechanism is more potent than rubber particle bridging. Therefore, this work should be applicable to highly crosslinked epoxies as well as the ductile epoxies investigated in this work.

\section{ACKNOWLEDGEMENT}

This work was funded by the National Science Foundation (Materials Research Group DMR-8708405).

\section{REFERENCES}

1 McGarry, F. J. Proc. R. Soc. London, Ser. A 1970, 59, 68

2 Sultan, J. N., Laible, T. C. and McGarry, F. J. Appl. Polym. Symp. 1971, 16, 127

3 Sultan, J. N. and McGarry, F. J. Polym. Eng. Sci. 1973, 13, 29

4 Meeks, A. C. Polymer 1974, 15, 675

5 Drake, R. and Siebert, A. SAMPE Quarterly 1975, July, 11

6 Riew, C. K., Rowe, E. H. and Siebert, A. Adv. Chem. Ser. 1976, 154, 326

7 Bascom, W. D., Cottington, R. L. and Timmons, C. O. Appl. Polym. Symp. 1977, 32, 165

8 Kunz-Douglass, S., Beaumont, P. W. R. and Ashby, M. F. J. Mater. Sci. 1980, 15, 1109

9 Kunz, S. C. and Beaumont, P. W. R. J. Mater. Sci. 1981, 16, 3141

10 Bascom, W. D., Ting, R. Y., Moulton, R. J., Riew, C. K. and Siebert, A. R. J. Mater. Sci. 1981, 16, 2657

11 Kinloch, A. J., Shaw, S. J., Tod, D. A. and Hunston, D. L. Polymer 1983, 24, 1341

12 Kinloch, A. J., Shaw, S. J. and Hunston, D. L. Polymer 1983, 24, 1355

13 Sayre, J. A., Kunz, S. C. and Assink, R. A. Polym. Mater. Sci. Eng. 1983, 49, 442

14 Maxwell, D., Young, R, J. and Kinloch, A. J. J. Mater. Sci. Lett. $1984,3,9$
15 Yee, A. F. and Pearson, R. A. J. Mater. Sci. 1986, 21, 2462

16 Pearson, R. A. and Yee, A. F. J. Mater. Sci. 1986, 21, 2475

17 Pearson, R. A. and Yee, A. F. J. Mater. Sci. 1989, 24, 2571

18 Kinloch, A. J., Finch, C. A. and Hashemi, S. Polym. Commun. $1987,28,322$

19 Levita, G. Polym. Prepr. 1988, 29, 175

20 Bradley, W. L. Polym. Prepr. 1988, 29, 179

21 Pearson, R. A. and Yee, A. F. J. Mater. Sci. 1991, 26, 3828

Bucknall, C. B. and Partridge, I. K. Polym. Eng. Sci. 1986, 26, 54 Hedrick, J. L., Yilgor, I., Wilkes, G. L. and McGrath, J. E. Polym. Bull. 1985, 13, 201

Cecere, J. A. and McGrath, J. E. Polym. Prepr. 1986, 27, 299 Raghava, R. S. J. Polym. Sci. (B) Polym. Phys. 1988, 26, 65 Fu, Z. and Sun, Y. Polym. Prepr. 1988, 29, 177

Bucknall, C. B. and Gilbert, A. H. Polymer 1989, 30, 213

Kim, S. C. and Brown, H. R. J. Mater. Sci. 1987, 22, 2589

Ahmad, Z. B., Ashby, M. F. and Beaumont, P. W. R. Scr. Mettal. $1986,20,843$

Rose, L. R. F. Mech. Mater. 1987, 8, 11

Lange, F. F. Phil. Mag. 1971, 22, 983

Evans, A. G. Phil. Mag. 1972, 26, 1327

Lange, F. F. and Radford, K. C. J. Mater. Sci. 1971, 6, 1197

Kinloch, A. J., Maxwell, D. and Young, R. J. J. Mater. Sci. Lett. $1985,4,1276$

35 Farber, K. T. and Evans, A. G. Acta Mettal. 1983, 31, 565

36 Sue, H. J., Pearson, R. A. and Yee, A. F. Polym. Eng. Sci. 1991, 31, 793

Pearson, R. A. and Yee, A. F. J. Appl. Polym. Sci. 1993, 48, 1051 Brown, W. F. and Srawley, J. E. ASTM STP 410, 1966

Bucknall, C. B. 'Toughened Plastics', Applied Science, Barking, Essex, 1977

40 Maxwell, M. A. and Yee, A. F. Polym. Eng. Sci. 1981, 21, 205

41 Sue, H. J., Pearson, R. A., Parker, D. S., Huang, J. and Yee, A. F. Polym. Prepr. 1988, 30, 147

Hutchinson, J. W. Acta. Metall. 1987, 35, 1605

Ortiz, M. Trans. ASME 1987, 54, 54

Evans, A. G. and Faber, K. T. J. Am. Ceram. Soc. 1981, 64, 394

Gao, F. and Wang, T. J. Mater. Sci. Lett. 1990, 9, 1409

Sue, H. J. and Yee, A. F. J. Mater. Sci. 1989, 24, 1447

Kim, J. and Robertson, R. Polym. Mater. Sci. Eng. 1990, 63, 301 